\title{
Prevalence of metabolic syndrome among ethnic groups in China
}

\author{
Xuzhen Qin ${ }^{1 \dagger}$, Ling Qiu ${ }^{1 \dagger}$, Guodong Tang ${ }^{2+}$, Man-Fung Tsoi ${ }^{3}$, Tao Xu ${ }^{4}$, Lin Zhang ${ }^{1}$, Zhihong Qi ${ }^{1}$, \\ Guangjin Zhu ${ }^{5}$ and Bernard M. Y. Cheung ${ }^{3^{*}}$ (i)
}

\begin{abstract}
Background: Metabolic syndrome (MetS) is common in China, which has a multi-ethnic population of 1.3 billion. We set out to determine the prevalence of MetS and its components in different ethnic groups.

Methods: This nationwide cross-sectional survey involved 24,796 participants from eight ethnicities in six provinces in China from 2008 to 2011. MetS was defined using the modified National Cholesterol Education Program Adult Treatment Panel III criteria. Results were analysed using SPSS version 22.0 in 2018. Logistic regression was used for deriving odds ratios and $95 \%$ confidence intervals of risk factors for the MetS.

Results: The prevalence of MetS increased with age from 3.60\% to $21.68 \%$. After age standardization, the prevalence of MetS, in descending order, was 35.42\% (Korean), 22.82\% (Hui), 19.80\% (Han), 13.72\% (Miao), 12.90\% (Tujia), 12.04\% (Li), 11.61\% (Mongolian), 6.17\% (Tibetan). Korean ethnicity was associated with a higher prevalence in five components of MetS, while Tibetan ethnicity was associated with lower prevalence except decreased HDL cholesterol. Logistic regression analyses showed that age, drinking and being non-Tibetan were associated with a higher risk of MetS.

Conclusions: Within one country, albeit a large one, the prevalence of MetS can vary greatly. Chinese of Korean ethnicity had a much higher prevalence than Tibetan ethnicity. Measures to tackle MetS should be tailored to the ethnic groups within a population.
\end{abstract}

Keywords: Metabolic syndrome, Ethnic group, China

\section{Background}

Metabolic Syndrome (MetS) is a cluster of related abnormalities that include abdominal obesity, insulin resistance, dyslipidaemia and elevated blood pressure [1,2]. The National Cholesterol Education Program's Adult Treatment Panel III (NCEP: ATPIII), World Health Organization (WHO) and International Diabetes Federation (IDF) use this syndrome to highlight the risk of patients developing cardiovascular disease (CVD) and type 2 diabetes (T2DM) $[1,3]$. We previously reported that the prevalence of MetS

\footnotetext{
* Correspondence: mycheung@hku.hk

${ }^{+}$Xuzhen Qin, Ling Qiu and Guodong Tang contributed equally to this work.

${ }^{3}$ Department of Medicine, University of Hong Kong, Queen Mary Hospital,

102 Pokfulam Road, Hong Kong, China

Full list of author information is available at the end of the article
}

in the United States was $33.6 \%$ in the adult population [4]. MetS predicts the development of diabetes [5] and hypertension [6], and is also associated with coronary artery disease (CAD) [7] and increased mortality [8].

While the natural history of MetS and how it develops have been well described in Hong Kong Chinese, a large number of observational studies have also been conducted in China, the population of which is mostly of Han ethnicity $[9,10]$. A meta-analysis showed that the pooled prevalence of MetS in China was 24.5\% among subjects aged over 15 years. Individuals living in urban areas had a higher risk of having MetS than those living in rural areas [11]. However, China is a multi-ethnic country with at least 55 ethnic groups. There are few studies focused on 
MetS among different ethnic groups in China, and most of these studies focused on a single ethnic group [7, 12, 13]. The heterogeneity in study design, inclusion criteria and definition of MetS makes it difficult to compare the prevalence in different ethnic groups.

Building on the Expansion Investigation of Human Physiology Constant Study in China [14], we made use of unified inclusion criteria and centralized measurements to determine the prevalence of Metabolic Syndrome (MetS) among different ethnicities in six provinces of China using the modified NCEP: ATPIII criteria [15].

\section{Methods}

\section{Description of the study}

The Expansion Investigation of Human Physiology Constant was a nationwide cross-sectional, random and multistage clustering sampled survey in China that is part of the National Constitution and Health Database in 2008-2011 [16]. Our previous studies based on the National Constitution and Health Database had shown several factors associated with cardiovascular disease [14, 17]. This study was expanded to six provinces and eight ethnic groups according to the geographical, ethnical and economic characteristics from the previous studies. All study personnel was trained with standardised working manuals and surveys before they conducted this study. Sample processing, testing and quality control were conducted by certified personnel in a central laboratory (Department of Laboratory Medicine in Peking Union Medical College Hospital) to ensure the consistency and stability of the measurements.

\section{Subjects}

Participants aged 8-86 years were recruited from six provinces (Inner Mongolian, Heilongjiang, Ningxia, Hunan, Yunnan, Sichuan) using a random, multistage clustersampling method as shown in Fig. 1. Eight ethnic groups, including Han, Mongolian, Korean, Hui, Miao, Li, Tibetan and Tujia, were enrolled in this study. Inclusion criteria were absence of self-reported systemic diseases, such as CAD, renal disease, autoimmune disease, hypersensitivity, gastrointestinal disease, pulmonary disease, and cancer. Considering different customs of ethnic groups, we included participants aged 8-86. Exclusion criteria included significant abnormalities on physical examination, and

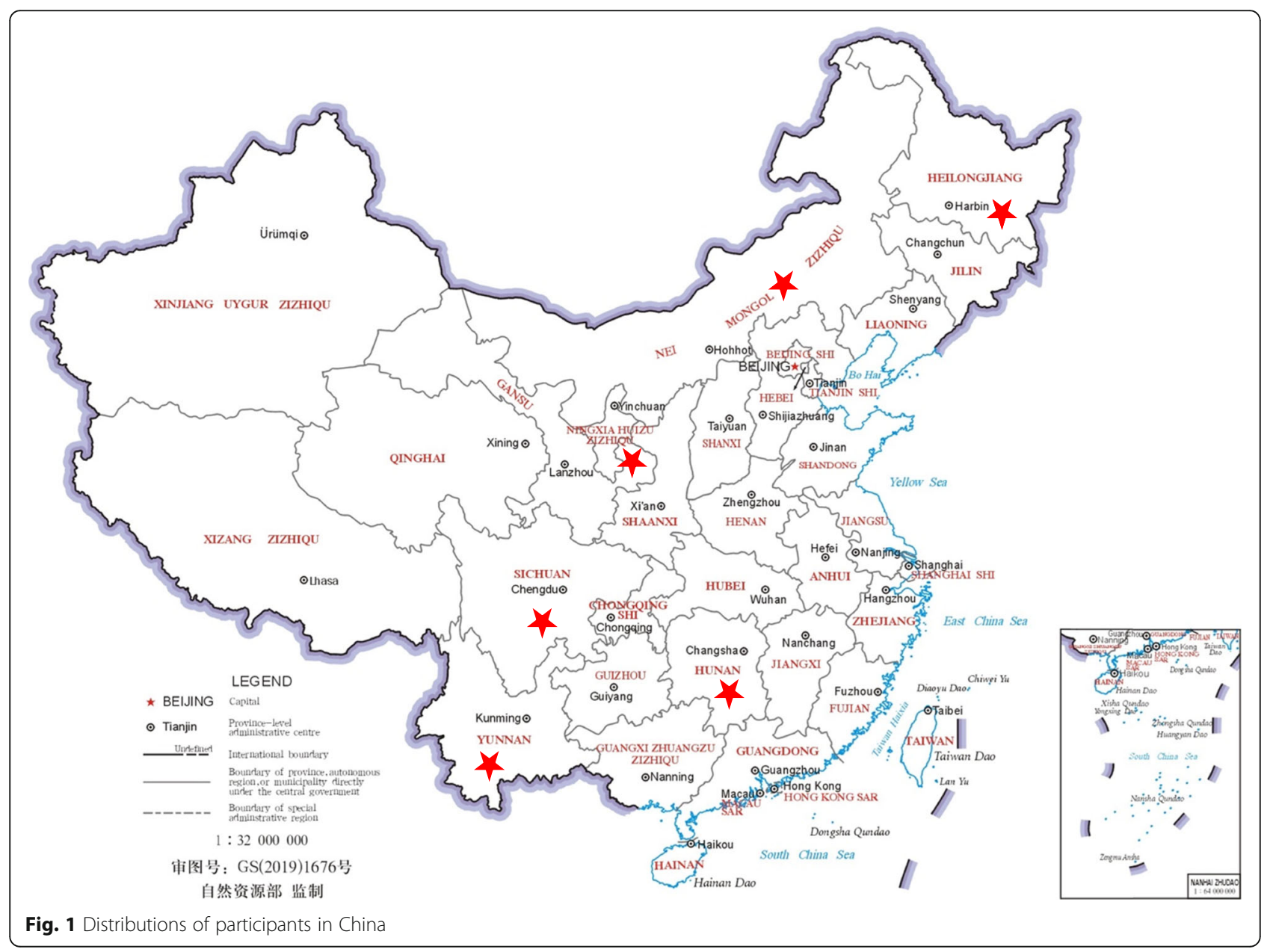


having a fever, acute illness, or hospitalization within 15 days. This study was approved by the Ethics Committee of Institute of Basic Medical Sciences Chinese Academy of Medical Sciences (No.005-2008). Written consents were obtained from all participants.

\section{Data collection}

Participants were required to complete a demographic questionnaire and a health behaviour questionnaire, which included smoking, alcohol consumption and physical activity. Trained personnel assisted the participants in completing the questionnaires.

\section{Measurements}

All participants were asked to avoid smoking and heavy physical activity for at least $2 \mathrm{~h}$ before the physical examinations, which included resting blood pressure, height, weight, and waist circumference (WC). Two blood pressure measurements were taken using OMRON HEM7000 electronic sphygmomanometer (OMRON HealthCare, Kyoto, Japan) after the participants had rested in a sitting position for at least $5 \mathrm{~min}$.

Participants were required to fast for 8 to $12 \mathrm{~h}$ before blood sampling. Fasting blood glucose (GLU), total cholesterol, triglycerides, high-density lipoprotein cholesterol (HDL cholesterol), and low-density lipoprotein cholesterol (LDL cholesterol) were analysed using a Hitachi 7020 chemistry analyser (Hitachi, Tokyo, Japan). Body mass index (BMI) was a person's weight in kilograms divided by the square of height in meters.

\section{Definition of metabolic syndrome}

The modified ATP III criteria were applied in the diagnosis of MetS, which requires the presence of at least three out of five factors [15]: (i) Abdominal density as defined by waist circumference $\geq 90 \mathrm{~cm}$ in men and $\geq 80$ $\mathrm{cm}$ in women; (ii) triglycerides $\geq 1.7 \mathrm{mmol} / \mathrm{L}$; (iii) $\mathrm{HDL}$ cholesterol $<1.03 \mathrm{mmol} / \mathrm{L}$ in men and $<1.29 \mathrm{mmol} / \mathrm{L}$ in women; (iv) systolic blood pressure (SBP) $\geq 130 \mathrm{mmHg}$ or diastolic blood pressure (DBP) $\geq 85 \mathrm{mmHg}$; (v) GLU $\geq 5.6 \mathrm{mmol} / \mathrm{L}$ as impaired fasting glucose (IFG).

\section{Statistical analysis}

Results were analysed using SPSS version 22.0 (IBM SPSS Statistics, Armonk, NY, USA) in 2018. Descriptive statistics were expressed as frequency (percentage) for categorical data or mean \pm SD or median (interquartile range) for continuous variables. Missing data were less than $3 \%$ for all included variables. Continuous variables were compared among groups using one-way ANOVA and Kruskal-Wallis tests as appropriate. Categorical variables were compared among groups using Chi-square test. Logistic regression was used to identify the predictors for MetS. Gender, age, ethnicities, exercises, smoking status, drinking status were included in the model. Age groups and gender were entered into the first adjusted model. Other predictor variables were entered stepwise if $P<0.05$ and removed if $P>$ $0 \cdot 10$. Exercise was added as a covariate in the second adjusted model and ethnicities, drinking status, smoking status were applied in the third adjusted model. Odds ratios (ORs) and 95\% confidential interval (95\% CI) were estimated. A two-tailed $P<0.05$ was considered statistically significant. Data from the Sixth National Population Census of the People's Republic of China provided by the National Statistics Bureau of China were used as the standard population. Age-specific prevalence and age-standardized prevalence were estimated from the standard population.

\section{Results}

A total of 24,796 participants were enrolled in this study (Fig. 2). The characteristics are summarized in Supplementary Table 1 . There was a significant difference in the median age among participants from different provinces and ethnic groups (shown in Supplementary Table 1, 2 and 3). Compared with Hans in the same province, participants from Tibetan, Miao, Tujia, Mongolian, Li ethnic groups were younger $(P<0 \cdot 001)$.

\section{The prevalence of MetS in eight ethnic groups of China}

The age, gender and ethnic-specific crude and standardised prevalence of MetS are summarised in Table 1. The prevalence of MetS in males (13.5\%) was slightly higher than in females $(12.6 \%)(P=0.28)$. The prevalence of MetS was substantially higher above age $25(24.7 \%)$ compared with age $\leq 25(2.3 \%)(P<0.001)$. There was a significant difference in MetS prevalence among ethnic groups $(P<0.001)$. Korean Chinese had the highest prevalence of 35.42 , followed by Hui, Han, Miao, Tujia, Li, Mongolian and Tibetan (Table 1, Fig. 3).

\section{The prevalence of MetS components}

Figure 4 shows the prevalence of components of MetS in different ethnic groups. The highest prevalence of abdominal obesity, hyperlipidaemia, decreased HDL cholesterol, elevated blood pressure and IFG were found in Han, Li, Hui, Li and Korean ethnicities, respectively;

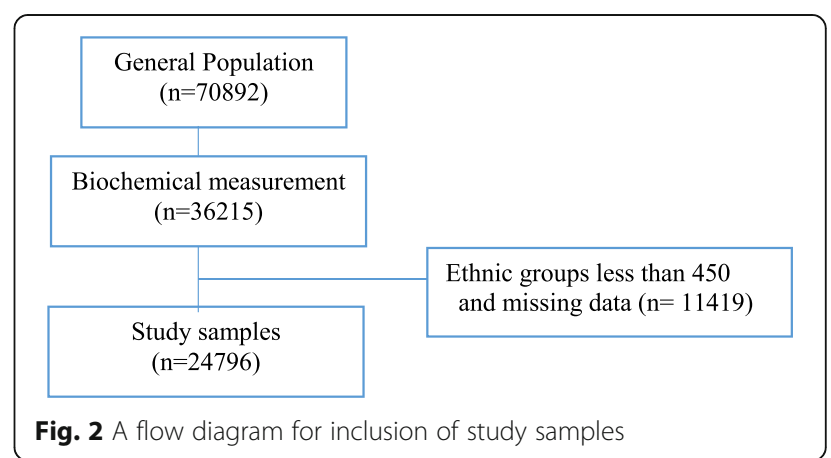




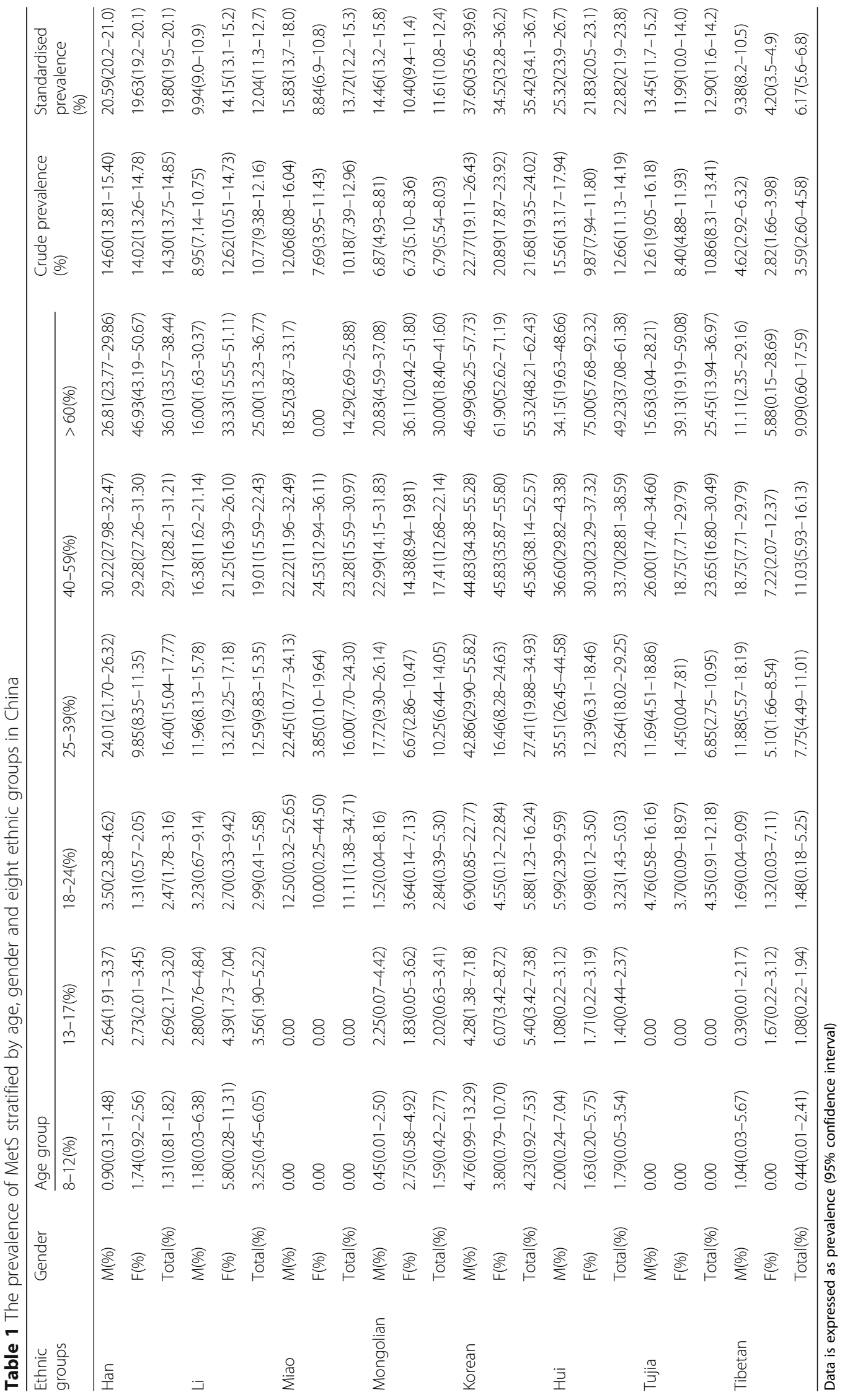




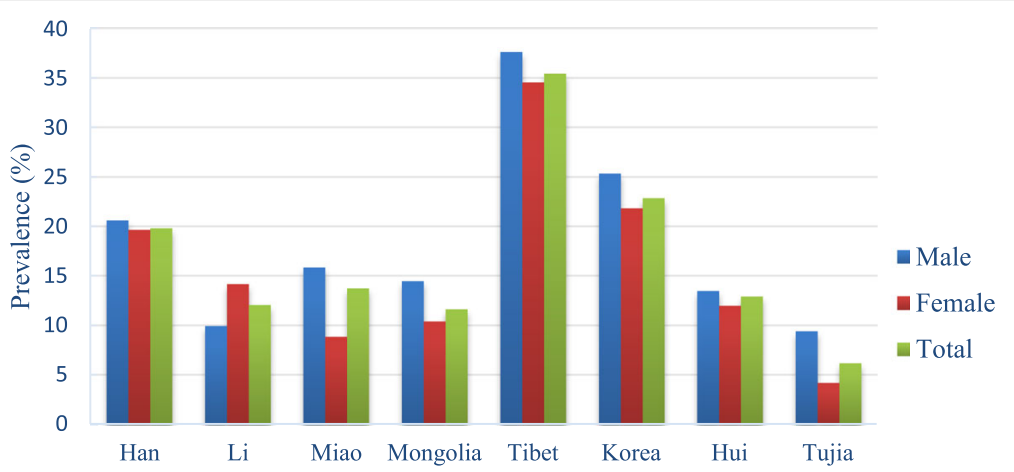

Figure 3 Standardized prevalence of MetS in ethnic groups in China stratified by gender

Fig. 3 Standardized Prevalence of MetS in ethnic groups in China stratified by gender

while the lowest prevalence of the components of MetS appeared in $\mathrm{Li}$ and Tibetan ethnicities (Fig. 4). In all ethnic groups, the prevalence of decreased HDL cholesterol and elevated blood pressure were the two highest among the five components, while IFG was less prevalent, especially in Miao, Mongolian, Tibetan, Hui and Tujia ethnicities.

\section{Predictors for MetS}

Predictors for MetS are summarized in Table 2. Age, gender, smoking, alcohol consumption and ethnic groups were all significant risk factors for MetS in the crude model $(p<$ $0.001, p=0.027, p<0.001, p<0.001, p<0.001$, respectively). Compared to females, males had an increased risk of MetS [OR (95\% CI): 1.087 (1.009-1.171), $p=0.027]$. However, maleness [1.028 (0.930-1.137), $p=0.059]$ and smoking [0.908 (0.806-1.022), $p=0.110]$ were not significant factors for MetS after multivariable adjustment. Compared to participants aged 8 to 12 years, the OR increased with age, and was highest in participants aged 60 to 86 years [34.117 (24.673-47.177), $p<0.001$ ]. Relative to Tibetans, all other ethnicities were associated with increased risk of MetS.
Korean Chinese had the highest adjusted risk for MetS [5.989 (4.249-8.442), $p<0.001]$, followed by Hui [4.020 (2.859-5.653), $p<0.001]$, Han [2.975 (2.194-4.034), $p<$ $0.001]$, Li [2.096 (1.487-2.954), $p<0.001]$, Miao [1.961 (1.262-3.048), $p=0.003$ ], Mongolian [1.835 (1.272-2.648), $p=0.001]$ and Tujia [1.753 (1.166-2.637), $p=0.007]$ ethnicities. MetS was not significantly associated with exercise in both crude and adjusted models $(P=0.140, P=0.710$, $P=0.710, P=0.905$, respectively).

\section{Discussion}

This study is the first large-scale multi-ethnic investigation of the prevalence of MetS in China. With 24,796 participants, it had sufficient power to estimate and compare prevalence in subgroups. Such comparisons are also valid and reliable because we had standardised protocols, rigorous quality control and a central clinical laboratory for measurements. The large sample size, standardised measurements and the quality control allowed us to have accurate estimates of the prevalence of MetS in the general population in China. It is a

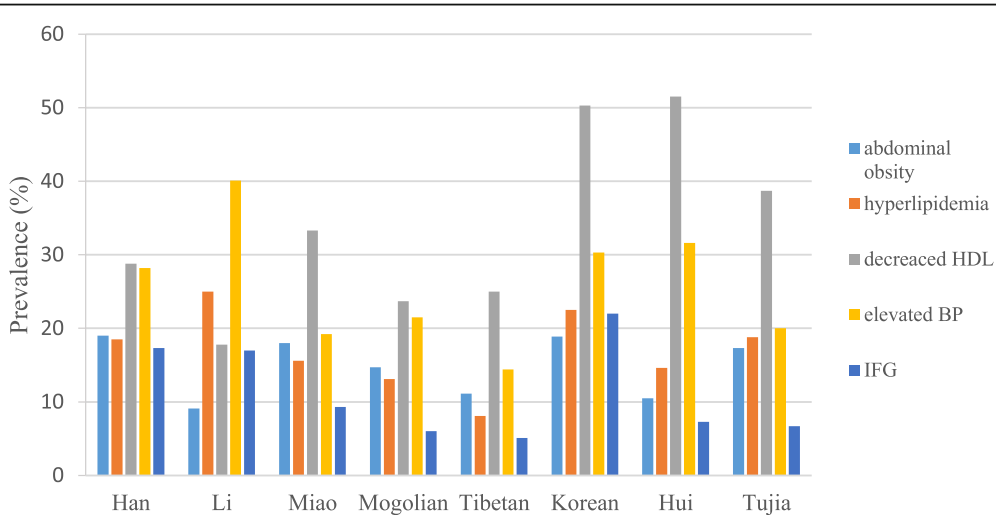

Fig. 4 The prevalence of MetS components in ethnic groups in China 
Table 2 Predictors of metabolic syndrome

\begin{tabular}{|c|c|c|c|c|c|}
\hline \multicolumn{2}{|l|}{ Parameters } & Crude model & Adjusted model 1 & Adjusted model 2 & Adjusted model 3 \\
\hline \multicolumn{2}{|l|}{$\overline{\text { Male }}$} & $1.087(1.009-1.171)$ & $1.110(1.024-1.202)$ & $1.110(1.025-1.203)$ & $1.028(0.930-1.137)$ \\
\hline \multicolumn{2}{|l|}{ Exercise } & $0.941(0.867-1.020)$ & $1.017(0.931-1.110)$ & $1.017(0.931-1.110)$ & $1.006(0.911-1.111)$ \\
\hline \multicolumn{2}{|l|}{ Smoking Status } & $1.867(1.702-2.048) \wedge$ & $0.977(0.873-1.093)$ & $0.979(0.875-1.096)$ & $0.908(0.806-1.022)$ \\
\hline \multicolumn{2}{|l|}{ Drinking Status } & $2.159(1.975-2.360) \wedge$ & $1.322(1.186-1.472) \wedge$ & $1.320(1.184-1.470) \wedge$ & $1.348(1.203-1.509) \wedge$ \\
\hline \multirow[t]{6}{*}{ Age groups } & $8-12$ & 1 (reference) & 1 (reference) & 1 (reference) & 1 (reference) \\
\hline & $13-17$ & $1.767(1.282-2.435)$ & $1.770(1.285-2.440) \wedge$ & $1.769(1.283-2.438) \wedge$ & $1.712(1.208-2.425)$ \\
\hline & $18-24$ & $1.870(1.306-2.677)$ & $1.867(1.304-2.674)$ & $1.863(1.301-2.669)$ & $1.727(1.176-2.535)$ \\
\hline & $25-39$ & $12 \cdot 191(9.090-16.350) \wedge$ & $12.242(9.128-16.418) \wedge$ & $12.238(9.125-16.414) \wedge$ & $11.67(8.452-16.113) \wedge$ \\
\hline & $40-59$ & $26 \cdot 041(19 \cdot 516-34 \cdot 747) \wedge$ & $26 \cdot 16(19.605-34.907) \wedge$ & $26 \cdot 162(19.606-34.910) \wedge$ & $24.583(17.892-33.776) \wedge$ \\
\hline & $60-86$ & $38.350(28.512-51.584) \wedge$ & $38.225(28.418-51.416) \wedge$ & $38.176(28.38-51.354) \wedge$ & $34.117(24.673-47 \cdot 177)$ \\
\hline \multirow[t]{8}{*}{ Ethnic groups } & Tibetan & 1 (reference) & 1 (reference) & 1 (reference) & 1 (reference) \\
\hline & Han & $4.482(3.358-5.982) \wedge$ & $2.917(2.165-3.930) \wedge$ & $2.916(2.165-3.929) \wedge$ & $2.975(2.194-4.034) \wedge$ \\
\hline & $\mathrm{Li}$ & $3.243(2 \cdot 356-4 \cdot 465) \wedge$ & $1.991(1.431-2.771) \wedge$ & $1.984(1.417-2.778) \wedge$ & $2.096(1.487-2.954) \wedge$ \\
\hline & Miao & $3.043(2.004-4.620) \wedge$ & $1.877(1.213-2.903)$ & $1.877(1.214-2.904)$ & $1.961(1.262-3.048)$ \\
\hline & Mongolian & $1.955(1.382-2.765) \wedge$ & $1.815(1.266-2.602)$ & $1.815(1.266-2.602)$ & $1.835(1.272-2.648)$ \\
\hline & Korean & $7.436(5 \cdot 419-10 \cdot 205) \wedge$ & $6.116(4.377-8.547) \wedge$ & $6.112(4.373-8.543) \wedge$ & $5.989(4.249-8.442) \wedge$ \\
\hline & Hui & $3.893(2.835-5.345) \wedge$ & $3.629(2.605-5.054) \wedge$ & $3.625(2.602-5.052) \wedge$ & $4.020(2.859-5.653) \wedge$ \\
\hline & Tujia & $3.271(2 \cdot 219-4 \cdot 824) \wedge$ & $1.721(1.151-2.575)$ & $1.722(1.151-2.576)$ & $1.753(1.166-2.637)$ \\
\hline
\end{tabular}

Data is expressed as odds ratio (95\% confidence interval)

$\wedge p<0.001$

Adjusted model 1: adjusted for gender, age groups

Adjusted model 2: adjusted for gender, age groups and exercises

Adjusted model 3: adjusted for gender, age groups, exercises, ethnicities, smoking status and drinking status

strength of the study to recruit from the age of 8 to 86 , which is an uncommonly wide range for an epidemiological study. The study did not recruit subjects below the age of 8 or above the age of 86 for practical reasons.

The prevalence of MetS in Hans in the present study was higher than a previous national study in China [18], but lower or similar to the prevalence in US and Europe $[19,20]$. Due to economic development, consumption of dairy products and fast foods had doubled [21, 22], which might have contributed to the increasing trend in MetS. The prevalence of MetS was slightly higher in men in this study, in contrast to some other populations [23].

The prevalence of MetS in Korean, Hui and Mongolian Chinese in our study is relatively higher than previous studies' in these ethnicities [24-26]. A study using the Korean National Health and Nutrition Examination Survey (KNHANES) data from 2008 to 2013 reported that the prevalence of MetS among the Korean participants aged $\geq 20$ years was $28.9 \%$ according to the modified NCEP: ATPIII definition [25]. A study conducted among rural adults in Ningxia in 2008 reported that the age-adjusted prevalence of MetS was 13.7\% with International Diabetes Federation definition [26]. In Mongolians aged over 18 years, the prevalence of MetS in men was higher than in women $(36.7 \%$ vs. $17.8 \%)$ [24]. However, a study of Tibetan immigrants in India aged over 20 years reported a prevalence of $10.6 \%$ in men and $33.3 \%$ in women [27]. In our study, Tibetans had a much lower prevalence of MetS as a result of possible volunteer bias.

This study revealed that the prevalence of the components of MetS varied greatly in different ethnicities. These differences may be due to genetic factors and environmental factors, which include diet and lifestyle. A randomized dietary and behavioural interventional study showed that a Tibetan diet reduced body weight and BMI in patients with CAD and MetS [28]. This might be the reason why Tibetans in our study had a low prevalence of MetS. The increased prevalence of MetS in Korean ethnicity might also be explained by diet, since it has been shown that dietary intakes of total fat and saturated fatty acids were significantly associated with MetS [29].

The association of gender with MetS is controversial [30-32]. In this study, the association of maleness with MetS was weak and became not significant in the multivariable model. In previous studies, it have been shown that advanced age and drinking status were both associated with higher risk of MetS, while the association with exercise and smoking status was equivocal [33-35]. Our finding that Chinese of Korean and Tibetan ethnicities had the highest and lowest prevalence for MetS respectively should prompt further studies to explore the possible causes of ethnic difference in the risk of developing 
MetS. Such studies should include a detailed dietary and lifestyle survey, and a study of socioeconomic and genetic factors.

\section{Limitations}

There were some limitations in this study. China is a large country, so 24,796 participants represent only a small fraction of the total population. We were unable to study minorities in very remote parts of China. Different ethnic groups have different religious beliefs and customs, and these may account for some of the differences.

\section{Conclusions}

In this large multi-ethnic population-based survey, the age-standardized prevalence of MetS varied greatly in different ethnic groups, ranging from $6.18 \%$ to $35.43 \%$. Korean ethnicity was associated with a higher prevalence of MetS and its components, while Tibetan ethnicity was associated with a lower prevalence of MetS and its components except decreased HDL cholesterol.

\section{Supplementary information}

Supplementary information accompanies this paper at https://doi.org/10. 1186/s12889-020-8393-6.

Additional file 1: Table S1. Characteristics of included participants stratified by ethnic groups. Table S2. Characteristics of included participants stratified by provinces. Table S3. Region, ethnicity and gender specific characteristics of participants stratified by provinces.

\section{Abbreviations \\ BMI: Body mass index; CAD: Coronary artery disease; CVD: Cardiovascular disease; DBP: Diastolic blood pressure; GLU: Fasting blood glucose; HDL cholesterol: High-density lipoprotein cholesterol; IDF: World Health Organization (WHO) and International Diabetes Federation; IFG: Impaired fasting glucose; KNHANES: Korean National Health and Nutrition Examination Survey; LDL cholesterol: Low-density lipoprotein cholesterol; MetS: Metabolic syndrome; NCEP: ATPIII: The National Cholesterol Education Program's Adult Treatment Panel III; ORs, 95\% Cl: Odds ratios and 95\% confidential interval; SBP: Systolic blood pressure; T2DM: Type 2 diabetes; WC: Waist circumference}

\section{Acknowledgements}

We acknowledge the assistance of the late Prof. James Miller from the University of Louisville in manuscript preparation. QXZ was the recipient of a Dr. Cheng Yu Tung Fellowship. We declare that the abstract of this manuscript has been presented at a research conference, the Lancet-CAMS Health Summit, 2019.

\section{Authors' contributions}

QXZ and TGD performed statistical analysis, interpreted the data and wrote the first draft. MFT, XT, ZL, QZH and BMYC contributed to data analysis and the writing of the final version of the manuscript. QL and ZGJ designed the study. All authors have approved the final version of the manuscript and its conclusions.

\section{Funding}

This study was supported by grants from the Special Fund for Key Basic Research Project of the Ministry of Science and Technology of P.R. China (No. 2006FY110 300). The funding authority does not have roles in this study.

\section{Availability of data and materials}

The datasets used and/or analysed during the current study are available on reasonable request.

Ethics approval and consent to participate

We clarify the source of materials used in our study, and any permissions to collect such samples comply with institutional, national, or international guidelines. Written informed consent was obtained from participants or a parent or guardian for participants under 16 years old.

\section{Consent for publication}

Not applicable.

\section{Competing interests}

The authors declare that they have no competing interests.

\section{Author details}

'Department of Laboratory Medicine, Chinese Academy of Medical Sciences \& Peking Union Medical College Hospital, Beijing 100730, China.

2Department of Cardiology, Beijing Hospital of Health Ministry, Beijing 100730, China. ${ }^{3}$ Department of Medicine, University of Hong Kong, Queen Mary Hospital, 102 Pokfulam Road, Hong Kong, China. ${ }^{4}$ Department of Epidemiology and Statistics, Institute of Basic Medical Sciences, Chinese Academy of Medical Sciences \& School of Basic Medicine, Peking Union Medical College, Beijing 100005, China. ${ }^{5}$ Department of Pathophysiology, Institute of Basic Medical Sciences, Chinese Academy of Medical Sciences \& School of Basic Medicine, Peking Union Medical College, Beijing 100005, China.

\section{Received: 19 September 2019 Accepted: 24 February 2020}

\section{References}

1. Alberti KGMM, et al. The metabolic syndrome: a new worldwide definition. Lancet. 2005;366(9491):1059-62.

2. Cheung BM, Thomas GN. The metabolic syndrome and vascular disease in Asia. Cardiovasc Hematol Disord Drug Targets. 2007;7(2):79-85.

3. Expert Panel on Detection E, Treatment of High Blood Cholesterol in A. Executive summary of the third report of the National Cholesterol Education Program (NCEP) expert panel on detection, evaluation, and treatment of high blood cholesterol in adults (adult treatment panel III). JAMA. 2001;285(19):2486-97.

4. Cheung BM, et al. Prevalence of the metabolic syndrome in the United States National Health and nutrition examination survey 1999-2002 according to different defining criteria. J Clin Hypertens (Greenwich). 2006;8(8):562-70.

5. Cheung BM, et al. Development of diabetes in Chinese with the metabolic syndrome: a 6-year prospective study. Diabetes Care. 2007;30(6):1430-6.

6. Cheung BM, et al. Relationship between the metabolic syndrome and the development of hypertension in the Hong Kong cardiovascular risk factor prevalence Study-2 (CRISPS2). Am J Hypertens. 2008;21(1):17-22.

7. Gui MH, et al. Effect of metabolic syndrome score, metabolic syndrome, and its individual components on the prevalence and severity of angiographic coronary artery disease. Chin Med J. 2017;130(6):669-77.

8. Cheung BM, et al. Components of the metabolic syndrome predictive of its development: a 6-year longitudinal study in Hong Kong Chinese. Clin Endocrinol. 2008;68(5):730-7.

9. Gu D, et al. Prevalence of the metabolic syndrome and overweight among adults in China. Lancet. 2005;365(9468):1398-405.

10. Yang G. Salt intake in individuals with metabolic syndrome. Lancet. 2009; 373(9666):792-4

11. Li R, et al. Prevalence of metabolic syndrome in mainland China: a metaanalysis of published studies. BMC Public Health. 2016;16(1):296.

12. He J, et al. The optimal ethnic-specific waist-circumference cut-off points of metabolic syndrome among low-income rural Uyghur adults in far Western China and implications in preventive public health. Int J Environ Res Public Health. 2017;14(2):158

13. Yan YZ, et al. Association of Insulin Resistance with glucose and lipid metabolism: ethnic heterogeneity in far Western China. Mediat Inflamm. 2016;2016:1-8.

14. Qin $X$, et al. Association between gamma-glutamyltransferase and prehypertension. Mol Med Rep. 2012;5(4):1092-8. 
15. Grundy SM, et al. Diagnosis and management of the metabolic syndrome an American Heart Association/National Heart, Lung, and Blood Institute scientific statement. Circulation. 2005;112(17):2735-52.

16. Xu T, Zhu GJ, Han SM. Study of zero-inflated regression models in a largescale population survey of sub-health status and its influencing factors. Chin Med Sci J. 2017;32(4):218-25.

17. Wu J, et al. Prevalence and clustering of major cardiovascular risk factors in China: a recent cross-sectional survey. Medicine (Baltimore). 2016;95(10): e2712.

18. He Y, et al. Prevalence of metabolic syndrome and individual metabolic abnormalities in China, 2002-2012. Asia Pac J Clin Nutr. 2019;28(3):621-33.

19. Moore JX, Chaudhary N, Akinyemiju T. Metabolic syndrome prevalence by race/ethnicity and sex in the United States, National Health and nutrition examination survey, 1988-2012. Prev Chronic Dis. 2017;14:160287.

20. Agyemang C, et al. A cross-national comparative study of metabolic syndrome among non-diabetic Dutch and English ethnic groups. Eur J Pub Health. 2013;23(3):447-52.

21. Xue $\mathrm{H}$, et al. Time trends in fast food consumption and its association with obesity among children in China. PLoS One. 2016;11(3):e0151141.

22. Zhao Y, et al. Fast food consumption and its associations with obesity and hypertension among children: results from the baseline data of the childhood obesity study in China mega-cities. BMC Public Health. 2017; 17(1):933

23. Pucci G, et al. Sex- and gender-related prevalence, cardiovascular risk and therapeutic approach in metabolic syndrome: a review of the literature. Pharmacol Res. 2017;120:34-42

24. You $L$, et al. Prevalence of hyperuricemia and the relationship between serum uric acid and metabolic syndrome in the Asian Mongolian area. J Atheroscler Thromb. 2014;21(4):355-65.

25. Tran BT, Jeong BY, Oh JK. The prevalence trend of metabolic syndrome and its components and risk factors in Korean adults: results from the Korean National Health and nutrition examination survey 2008-2013. BMC Public Health. 2017:17(1):71.

26. Zhao $\mathrm{Y}$, et al. Prevalence of the metabolic syndrome among rural original adults in NingXia, China. BMC Public Health. 2010;10(1):140.

27. Lin BY, et al. The prevalence of obesity and metabolic syndrome in Tibetan immigrants living in high altitude areas in Ladakh, India. Obes Res Clin Pract. 2018;12(4):365-71.

28. von Haehling $\mathrm{S}$, et al. Weight reduction in patients with coronary artery disease: comparison of traditional Tibetan medicine and Western diet. Int $J$ Cardiol. 2013;168(2):1509-15.

29. Lee KW, et al. Association of dietary intakes of total and subtypes of fat substituted for carbohydrate with metabolic syndrome in Koreans. Endocr J. 2016:63(11):991-9.

30. Bentley-Lewis R, Koruda K, Seely EW. The metabolic syndrome in women. Nat Clin Pract Endocrinol Metab. 2007:3(10):696-704.

31. Vryonidou A, et al. MECHANISMS IN ENDOCRINOLOGY: metabolic syndrome through the female life cycle. Eur J Endocrinol. 2015;173(5):R153-63.

32. Ong KL, et al. Gender difference in blood pressure control and cardiovascular risk factors in Americans with diagnosed hypertension. Hypertension. 2008;51(4):1142-8.

33. Huang $\mathrm{JH}$, et al. Lifestyle factors and metabolic syndrome among workers: the role of interactions between smoking and alcohol to nutrition and exercise. Int J Environ Res Public Health. 2015;12(12):15967-78.

34. Yu M, et al. Associations of cigarette smoking and alcohol consumption with metabolic syndrome in a male Chinese population: a cross-sectional study. J Epidemiol. 2014;24(5):361-9.

35. Kwasniewska M, et al. Smoking status, the menopausal transition, and metabolic syndrome in women. Menopause. 2012;19(2):194-201.

\section{Publisher's Note}

Springer Nature remains neutral with regard to jurisdictional claims in published maps and institutional affiliations.

Ready to submit your research? Choose BMC and benefit from:

- fast, convenient online submission

- thorough peer review by experienced researchers in your field

- rapid publication on acceptance

- support for research data, including large and complex data types

- gold Open Access which fosters wider collaboration and increased citations

- maximum visibility for your research: over $100 \mathrm{M}$ website views per year

At $\mathrm{BMC}$, research is always in progress.

Learn more biomedcentral.com/submissions 